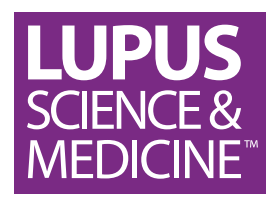

\title{
Risk of psychiatric disorders and all- cause mortality with belimumab therapy in patients with systemic lupus erythematosus: a meta-analysis of randomised controlled trials
}

\author{
Wenhui Xie (DD , ${ }^{1}$ Hong Huang, ${ }^{1}$ Siyan Zhan, ${ }^{2,3}$ Zhuoli Zhang ${ }^{1}$
}

To cite: Xie W, Huang $\mathrm{H}$, Zhan S, et al. Risk of psychiatric disorders and all-cause mortality with belimumab therapy in patients with systemic lupus erythematosus: a meta-analysis of randomised controlled trials. Lupus Science \& Medicine 2021;8:e000534. doi:10.1136/ lupus-2021-000534

- Additional supplemental material is published online only. To view, please visit the journal online (http://dx.doi.org/10. 1136/lupus-2021-000534).

Received 14 July 2021 Accepted 8 October 2021

D) Check for updates

(C) Author(s) (or their employer(s)) 2021. Re-use permitted under CC BY-NC. No commercial re-use. See rights and permissions. Published by BMJ.

${ }^{1}$ Department of Rheumatology and Clinical Immunology, Peking University First Hospital, Beijing, China

${ }^{2}$ Department of Epidemiology and Biostatistics, School of Public Health, Peking University, Beijing, China

${ }^{3}$ Research Center of Clinical Epidemiology, Peking University Third Hospital, Beijing, China

Correspondence to

Professor Zhuoli Zhang; zhuoli. zhang@126.com

\section{ABSTRACT}

Objectives To evaluate the risk of psychiatric disorders and all-cause mortality associated with belimumab therapy in patients with SLE.

Methods A literature search of four electronic bibliographic databases, including PubMed, EMBASE, Scopus and Cochrane databases, was conducted for randomised controlled trials (RCTs) reporting adverse reactions between belimumab and placebo. OR and 95\% Cl were calculated using the Mantel-Haenszel method with fixed-effects or random-effects model, depending on the heterogeneity test.

Results In total, 11 eligible RCTs including 8824 patients with SLE were randomised into belimumab (5160 patients with 5552 patient-years) and placebo (3664 patients with 3985 patient-years) groups, respectively. Overall, no increased risk was identified with belimumab therapy at all dosages compared with placebo in patients with SLE regarding all psychiatric disorders (OR $0.89,95 \% \mathrm{Cl}$ 0.64 to $1.23, I^{2}=58 \%$ ) and all-cause mortality (OR 1.10, $95 \% \mathrm{Cl} 0.64$ to $1.89, I^{2}=0 \%$ ). The subgroup analysis of psychiatric disorders also revealed no statistically elevated risks in serious psychiatric disorders (OR 1.15, 95\% Cl 0.77 to $1.70, \mathrm{I}^{2}=47 \%$ ), non-serious psychiatric disorders (OR $0.83,95 \% \mathrm{Cl} 0.60$ to $1.16, \mathrm{I}^{2}=52 \%$ ), suicidal ideation or behaviour (OR $0.87,95 \% \mathrm{Cl} 0.57$ to $1.33, \mathrm{I}^{2}=0 \%$ ), and depression (OR $1.29,95 \% \mathrm{Cl} 0.90$ to $1.85, \mathrm{I}^{2}=15 \%$ ). Secondary analysis restricting belimumab at approved dose of $10 \mathrm{mg} / \mathrm{kg}$ only yielded similar results.

Conclusion Belimumab therapy overall does not increase psychiatric events and all-cause mortality risks, whereas the results from Belimumab Assessment of Safety in SLE Study are suggestive of increased risk of psychiatric adverse events with belimumab exposure. Consequently, post-marketing data are needed to ascertain its psychiatric safety, especially serious mental disorders.

\section{INTRODUCTION}

Belimumab is a humanised monoclonal antibody that inhibits the activity of $B$ cell activating factor. Based on the promising efficacy and satisfactory safety in the pivotal clinical programmes of SLE, belimumab has been

\section{Key messages}

What is already known about this subject?

- Belimumab has been licensed worldwide for the treatment of SLE, but the risk of psychiatric disorders and all-cause mortality associated with belimumab therapy remains undetermined.

What does this study add?

- This is the first meta-analysis, based on the best available datasets, showing belimumab therapy is not associated with significantly increased risk of psychiatric events and mortality, relative to placebo.

How might this impact on clinical practice or future developments?

- Belimumab therapy is not associated with increased risk of psychiatric events and mortality, but in consideration of the result of Belimumab Assessment of Safety in SLE Study, post-marketing data are decidedly needed to ascertain the psychiatric safety of belimumab, especially serious mental disorders.

- Special caution is needed when initiating belimumab therapy in patients with existing mental problems, such as depression, suicidal ideation or behaviour until more information becomes available.

- The search of risk factors for developing serious mental disorders and the characteristics of mental disorders associated with belimumab are urgently required.

licensed worldwide for the treatment of adult as well as paediatric patients with active and autoantibody-positive SLE despite standard treatment. $^{12}$ Besides, the beneficial effects of belimumab on renal outcomes in patients with lupus nephritis were also demonstrated in the Belimumab International Study in Lupus Nephritis. ${ }^{3}$

Belimumab is the first biological agent approved for SLE treatment in more than 50 years. Nevertheless, in 2019, the Medicines and Healthcare products Regulatory Agency 
raised an alarm of increased risk of serious psychiatric events, such as depression, suicidal ideation or behaviour in patients with SLE receiving belimumab compared with those receiving placebo based on interim findings from a randomised trial of Belimumab Assessment of Safety in SLE (BASE). Very recently, the published data of BASE Study showed patients with SLE with belimumab exposure had increased risks of psychiatric disorders, including serious depression, treatment-emergent suicidality, and sponsor-adjudicated serious suicide or self-injury events. ${ }^{4}$ In a recent phase III, open-labelled continuation study for up to 7 years, favourable safety profiles and treatment response were observed in 142 Japanese and Korean patients with SLE receiving belimumab therapy, with median duration of belimumab exposure of 1171 days and a total belimumab exposure of 458.9 patientyears. Regarding the psychiatric disorders, one serious event of depression was reported ( 0.2 events/100 patientyears) and no completed suicides or suicide attempts were observed. ${ }^{5}$ But owing to the relatively low frequency of psychiatric event, it is difficult to assess the psychiatric impact of belimumab in patients with SLE based on an individual randomised controlled trial (RCT) unless an adequate population is available. To fill the gap, we performed this meta-analysis of RCTs to evaluate the risk of psychiatric disorders and all-cause mortality associated with belimumab treatment in patients with SLE.

\section{METHODS}

This article is reported in line with the Preferred Reporting Items for Systematic Reviews and MetaAnalyses. ${ }^{6}$ The methods were stipulated in a protocol that was registered with the PROSPERO (registration number: CRD42021234298).

\section{Data sources}

Four electronic bibliographic databases, including PubMed, EMBASE, Scopus and Cochrane databases, were searched without language restrictions from inception through 20 January 2021 and an updated search was conducted on 21 August 2021. Studies were identified using the search terms belimumab and synonyms. The details of search strategy are available in online supplemental appendix S1. The US National Institutes of Health Ongoing Trials Register (www.clinicaltrials.gov), major annual meetings in 2015-2020 and reference lists of all included studies were searched for additional trials.

\section{Study selection}

We included double-blind RCTs that reported adverse events in patients with SLE receiving belimumab or placebo during the randomised controlled phase. Exclusion criteria included non-randomised design, single-arm extension study, observational studies, case report, editorial, review or no adverse events reported. Two investigators (WX, HH) independently screened all titles and abstracts for potential inclusion. Discordances were resolved by a third experienced investigator (ZZ).

\section{Data extraction and outcome assessment}

Two review authors (WX, HH) independently extracted data and assessed the quality of selected studies. Data extraction included first author, publication year, patients' demographics, clinical characteristics and study outcomes. The Cochrane quality assessment tool was used to assess the quality of included RCTs. ${ }^{7}$

The primary outcome was the risk of all psychiatric disorders and all-cause mortality associated with belimumab at all dosages or approved dose of $10 \mathrm{mg} / \mathrm{kg}$ compared with placebo. The secondary outcomes included serious psychiatric disorders, non-serious psychiatric disorders, suicidal ideation or behaviour, and depression.

\section{Statistical analysis}

To summarise the findings of the selected studies, the OR with $95 \%$ CI was calculated as an effect measure. Meta-analysis was performed using a Mantel-Haenszel random-effects or fixed-effects model according to heterogeneity between studies, assessed using the $\mathrm{I}^{2}$ statistic (low, $\mathrm{I}^{2}<25 \%$; moderate, 25\%-50\%; high, $\mathrm{I}^{2}>50 \%$ ). We conducted sensitivity analyses with the Peto method for the rare outcome and with exclusion of conference abstracts. Funnel plot analysis was used to detect the potential publication bias. For statistical significance, two-sided $\alpha$ was set at $p=0.05$. All data were recorded in a Microsoft Excel spreadsheet and analysed using Review Manager V.5.3 software (Cochrane Collaboration).

\section{RESULTS}

\section{Study selection and characteristics}

In total, 11 RCTs comprising 8824 patients with 9537 patient-years were included. The details of the study selection are summarised in figure 1. ${ }^{348-16}$ There were 5160 patients with 5552 patient-years and 3664 patients with 3985 patient-years randomised into belimumab and placebo groups, respectively. The included RCTs were mostly international multicentre studies, with a median observation period of 52 weeks, ranging from 15 to 104 weeks. All included studies were published in peerreviewed journals with full-text, except for one conference abstract. ${ }^{16}$ All studies were performed in adult SLE, except one study conducted in paediatric patients with SLE. ${ }^{14}$ Baseline characteristics of included patients were generally comparable regrading age, sex composition, disease duration and disease activity across most arms (online supplemental table S1).

Overall, the numbers of all psychiatric disorders and death during the controlled phase of these studies are summarised in table 1 . The crude incidence rates of psychiatric disorders and all-cause mortality in belimumab and placebo groups were 6.430, 0.576 and 5.094, 0.501 per 100 patient-years, respectively.

\section{All psychiatric disorders and all-cause mortality}

For comparison of belimumab against placebo, pooled analysis of 11 trials found no increased risk of all psychiatric disorders (OR $0.89,95 \%$ CI 0.64 to $1.23, \mathrm{I}^{2}=58 \%$ ) 


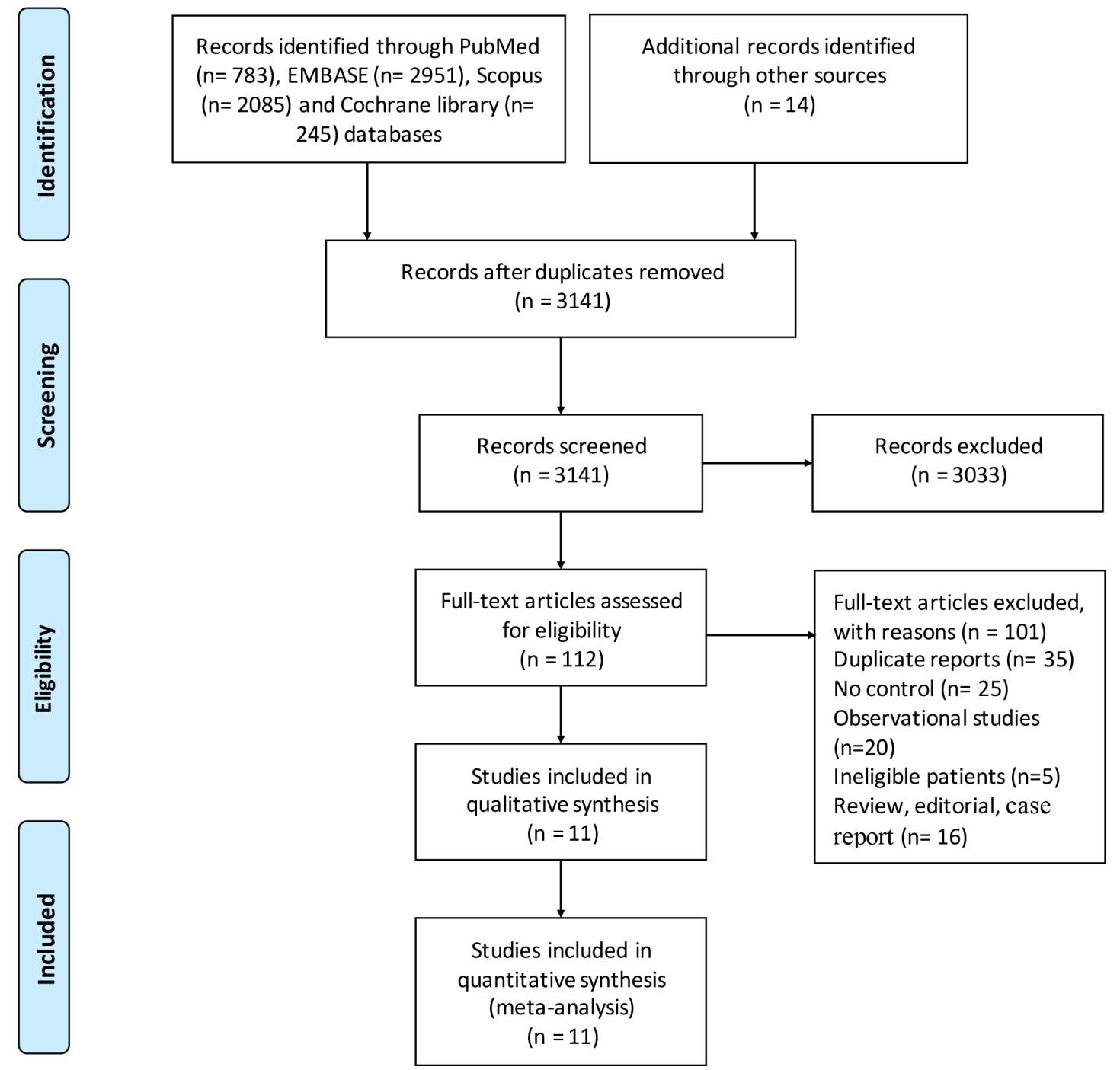

Figure 1 PRISMA flow diagram of study selection for systematic review and meta-analysis. PRISMA, Preferred Reporting Items for Systematic Reviews and Meta-Analyses.

and all-cause mortality (OR $1.10,95 \%$ CI 0.64 to 1.89 , $\mathrm{I}^{2}=0 \%$ ) with belimumab therapy at all dosages in general (figures 2 and 3). Furthermore, no statistical difference in all psychiatric disorders and all-cause mortality was observed between belimumab $10 \mathrm{mg} / \mathrm{kg}$ group and placebo group (all psychiatric disorders: OR $0.88,95 \% \mathrm{CI}$ 0.64 to $1.22, \mathrm{I}^{2}=54 \%$; all-cause mortality: OR $1.14,95 \% \mathrm{CI}$ 0.66 to $1.98, \mathrm{I}^{2}=0 \%$ ) (online supplemental table $\mathrm{S} 2$ ). In addition, the sensitivity analyses showed similar results using the Peto method for all-cause mortality $(<1 \%)$ (OR $1.19,95 \%$ CI 0.68 to $2.08, \mathrm{I}^{2}=0 \%$ ) or exclusively inclusion of full-text articles (all psychiatric disorders: OR 0.89, $95 \%$ CI 0.64 to $1.25, \mathrm{I}^{2}=62 \%$; all-cause mortality: OR 1.10 , $95 \%$ CI 0.64 to $1.89, \mathrm{I}^{2}=0 \%$ ) (online supplemental figure $1-3)$.

\section{Subgroup analysis of psychiatric disorders}

No significantly elevated risk was identified with belimumab exposure, relative to placebo, regarding serious psychiatric disorders (OR 1.15, 95\% CI 0.77 to 1.70, $\mathrm{I}^{2}=47 \%$ ), non-serious psychiatric disorders (OR 0.83 ,
$95 \%$ CI 0.60 to $1.16, \mathrm{I}^{2}=52 \%$ ), suicidal ideation or behaviour $\left(0.87,95 \%\right.$ CI 0.57 to $\left.1.33, \mathrm{I}^{2}=0 \%\right)$, and depression (OR $1.29,95 \%$ CI 0.90 to $1.85, \mathrm{I}^{2}=15 \%$ ) (figure 4 ). Of note, considering serious psychiatric disorders, suicidal ideation or behaviour, and depression as rare events $(<1 \%)$, we further applied Peto method and found no statistically increased risk (serious psychiatric disorders: OR $1.20,95 \%$ CI 0.80 to $1.79, \mathrm{I}^{2}=60 \%$; suicidal ideation or behaviour: OR $0.92,95 \%$ CI 0.59 to $1.43, \mathrm{I}^{2}=30 \%$; depression: OR $1.30,95 \%$ CI 0.92 to $1.83, \mathrm{I}^{2}=30 \%$ ) (online supplemental figure 4-6). Similar findings were also generated in the comparison of belimumab at $10 \mathrm{mg}$ / $\mathrm{kg}$ dose with placebo regarding the above-mentioned outcomes (online supplemental table S5).

\section{Risk of bias assessment}

Of the 11 included articles, 7 RCTs adequately reported the generation of random sequence and adequately described the concealed allocation. Blinding of participants, personnel and outcome assessor was also performed in seven RCTs. Incomplete data of outcome were well 


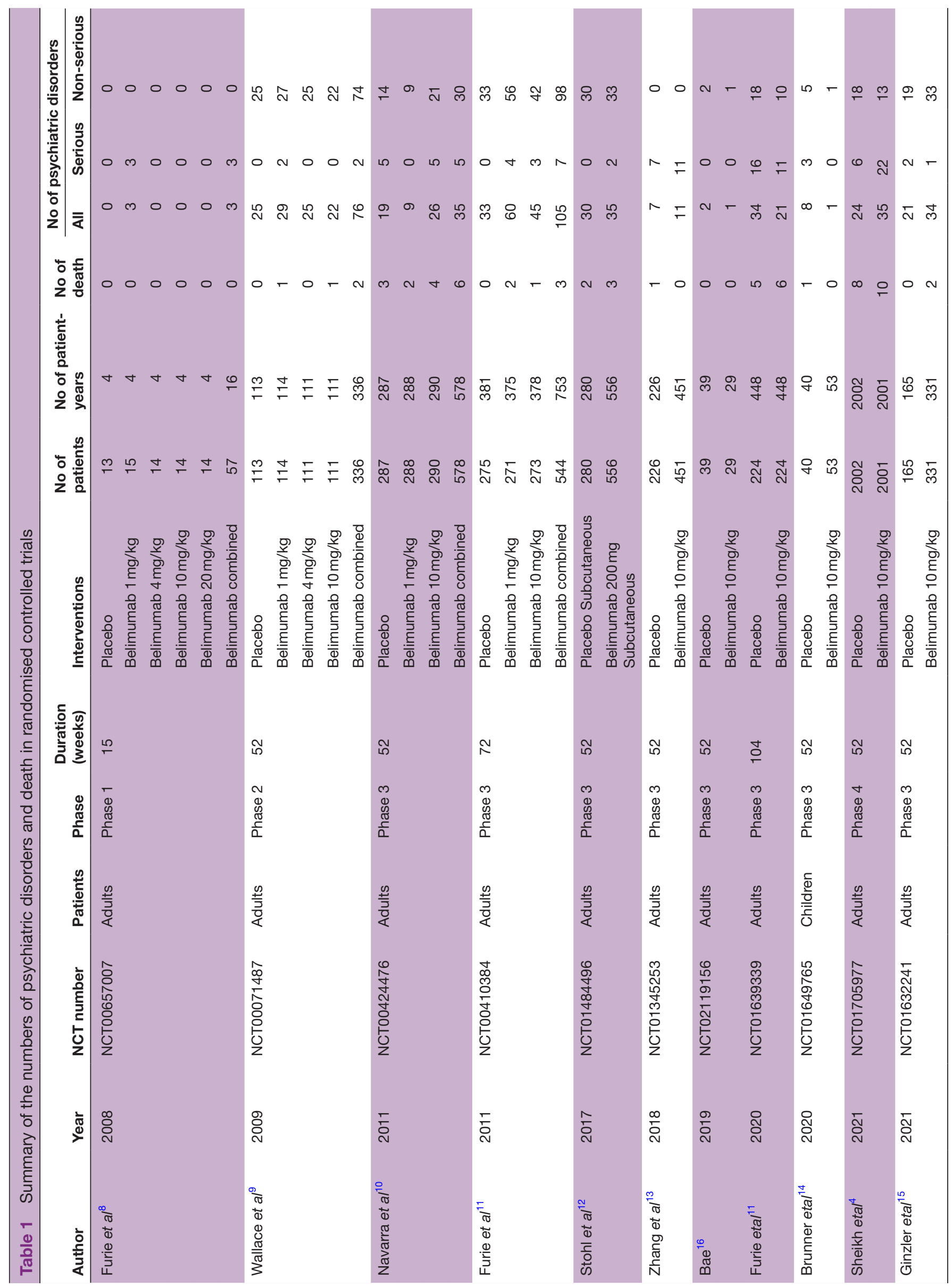




\begin{tabular}{|c|c|c|c|c|c|c|c|c|c|c|c|c|}
\hline Study or Subgroup & $\begin{array}{l}\text { Belimur } \\
\text { Events }\end{array}$ & $\begin{array}{l}\text { nab } \\
\text { Total }\end{array}$ & $\begin{array}{l}\text { Placek } \\
\text { Events }\end{array}$ & bo & Weight & $\begin{array}{l}\text { Odds Ratio } \\
\text { M-H, Random. } 95 \% \mathrm{Cl}\end{array}$ & Year & & $\begin{aligned} \text { Odds } \\
\text { M-H. Rando }\end{aligned}$ & Ratio & & \\
\hline Furie 2008 & 3 & 16 & 0 & 4 & $1.0 \%$ & $2.33[0.10,54.42]$ & 2008 & & & & & \\
\hline Wallace 2009 & 76 & 336 & 25 & 113 & $12.8 \%$ & $1.03[0.62,1.72]$ & 2009 & & & & & \\
\hline Furie 2011 & 105 & 753 & 33 & 381 & $14.4 \%$ & $1.71[1.13,2.58]$ & 2011 & & & $\because-$ & & \\
\hline Navarra 2011 & 35 & 578 & 19 & 287 & $11.8 \%$ & $0.91[0.51,1.62]$ & 2011 & & & & & \\
\hline Stohl 2017 & 35 & 556 & 30 & 280 & $12.8 \%$ & $0.56[0.34,0.93]$ & 2017 & & & & & \\
\hline Zhang 2018 & 11 & 451 & 7 & 226 & $7.1 \%$ & $0.78[0.30,2.05]$ & 2018 & & & & & \\
\hline Bae 2019 & 1 & 29 & 2 & 39 & $1.6 \%$ & $0.66[0.06,7.66]$ & 2019 & & & & & \\
\hline Brunner 2020 & 1 & 53 & 8 & 40 & $2.1 \%$ & $0.08[0.01,0.64]$ & 2020 & & & & & \\
\hline Furie 2020 & 21 & 448 & 34 & 448 & $12.0 \%$ & $0.60[0.34,1.05]$ & 2020 & & & & & \\
\hline Sheikh 2021 & 35 & 2001 & 24 & 2002 & $12.6 \%$ & $1.47[0.87,2.48]$ & 2021 & & & & & \\
\hline Ginzler 2021 & 34 & 331 & 21 & 165 & $11.8 \%$ & $0.78[0.44,1.40]$ & 2021 & & & & & \\
\hline Total $(95 \% \mathrm{Cl})$ & & 5552 & & 3985 & $100.0 \%$ & $0.89[0.64,1.23]$ & & & & & & \\
\hline Total events & 357 & & 203 & & & & & & & & & \\
\hline \multicolumn{8}{|c|}{ Heterogeneity: $\mathrm{Tau}^{2}=0.15 ; \mathrm{Chi}^{2}=23.61, \mathrm{df}=10(P=0.009) ; I^{2}=58 \%$} & \multicolumn{5}{|c|}{ 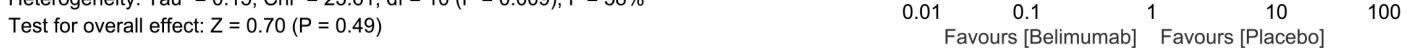 } \\
\hline
\end{tabular}

Figure 2 OR of all psychiatric disorders in patients with SLE receiving belimumab compared with placebo in randomised controlled trials.

balanced with no suggestion of selective outcome reporting in all included studies. Baseline characteristics of patients in all intervention groups were well balanced (figure 5). Funnel plot analysis showed no evidence of publication bias in all comparisons (online supplemental figure S7).

\section{DISCUSSION}

Belimumab has been approved for the treatment of adult and paediatric SLE, but there are residual uncertainties including psychiatric safety. ${ }^{1-3} 17$ Due to the variable psychiatric events observed in SLE clinical programmes, an additional BASE trial was required. The latest results of BASE Study indicated similar safety profiles between belimumab and placebo groups, except for higher frequency of hypersensitivity reactions and serious psychiatric events. ${ }^{4}$ To our knowledge, this is the first metaanalysis assessing the risk of psychiatric disorders and all-cause mortality associated with belimumab exposure. According to our results, belimumab treatment does not increase overall risks of psychiatric events and all-cause mortality relative to placebo. Subgroup analyses of psychiatric events revealed no significantly excess risk with belimumab exposure. Based on the best available datasets, this work reveals that belimumab is not associated with increased risk of psychiatric events and mortality. But in consideration of the results of BASE Study, further attention is needed to confirm the psychiatric safety. Due to the limited observational period in clinical trials and low frequency of serious psychiatric events, long-term observation in real-life setting is necessary to precisely measure such risks associated with belimumab therapy.

Currently, how belimumab triggers mental disorders is unclear. Further pharmacogenetic and pharmacogenomic investigations are required to explore the potential mechanism involved in psychiatric adverse effects associated with belimumab exposure. At moment, unravelling the characteristics of and risk factors for developing serious mental disorders associated with belimumab therapy should be performed either using pooled analysis of existing patient-level data in practice or using large sample of patients with SLE in future. Many practical problems need to be resolved, including the median (minimum, maximum) time from the start of belimumab therapy to the onset of the psychiatric event and the possibility of time-dependent effect of belimumab treatment. According to the results from the BASE Study, belimumab-treated patients who developed psychiatric events had longer SLE duration, high baseline SLE disease activity, and more past or current psychiatric disorder, as compared with placebo-treated patients who developed these events and overall patient population. ${ }^{4}$ These would

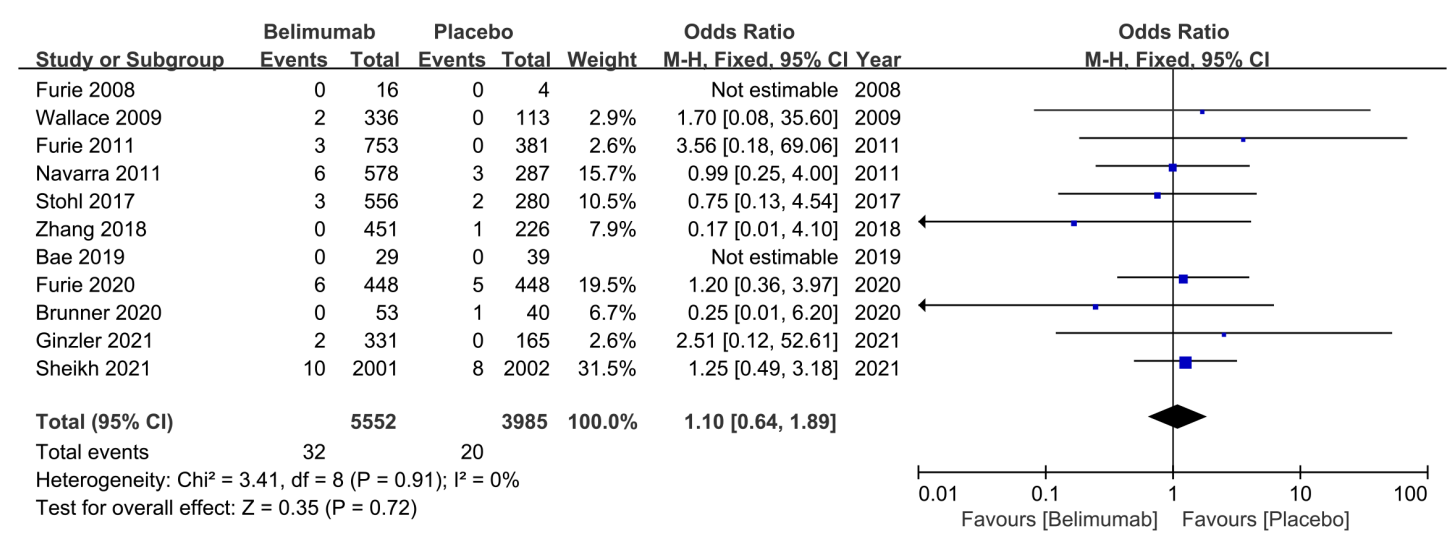

Figure 3 OR of all-cause mortality in patients with SLE receiving belimumab compared with placebo in randomised controlled trials. 
A

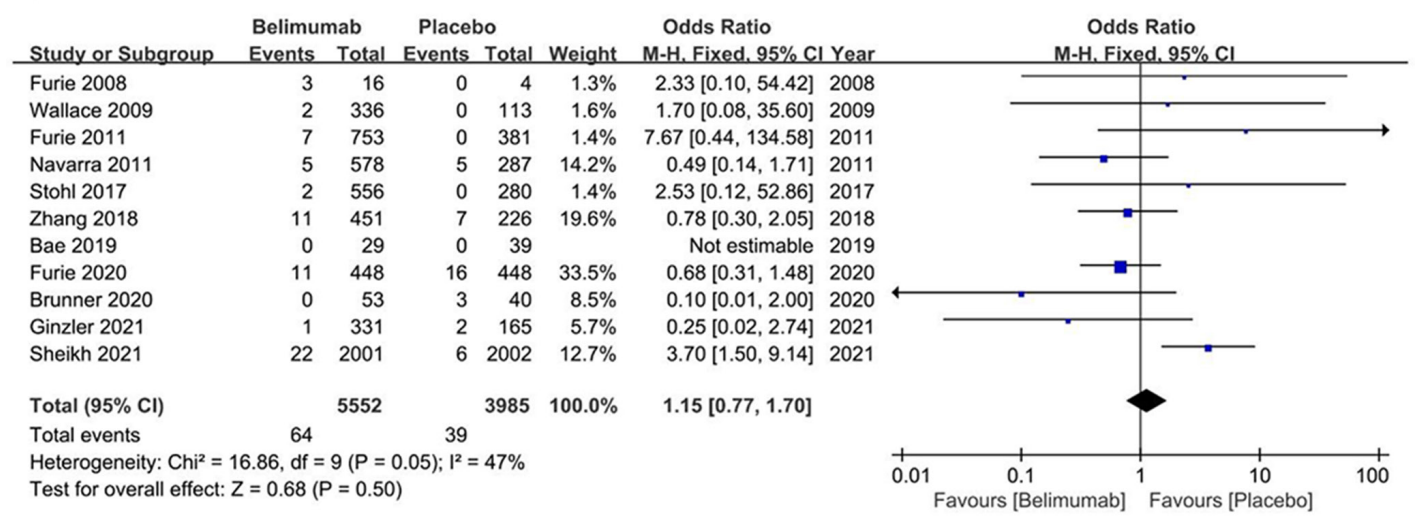

B

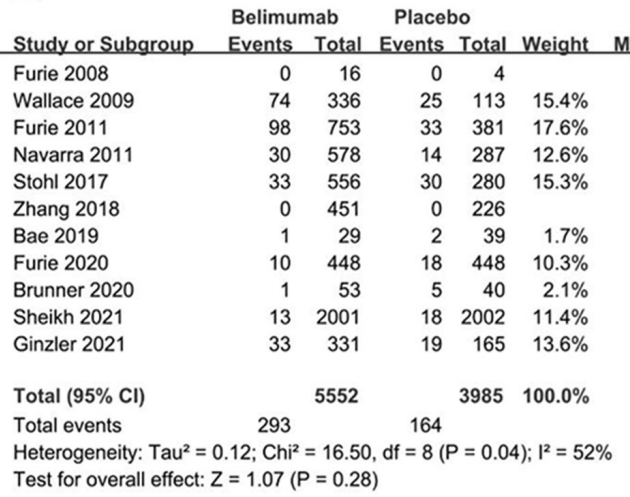

Odds Ratio

Odds Ratio

ndom. $95 \% \mathrm{Cl}$ Yea Not estimable 2008 $0.99[0.59,1.66] 2009$ $1.58[1.04,2.39] 2011$ $1.07[0.56,2.05] 2011$ $0.53[0.31,0.88] 2017$ Not estimable 2018 $0.66[0.06,7.66] 2019$ $0.55[0.25,1.20] 2020$ $0.13[0.02,1.20] 2020$ $0.72[0.35,1.47] 2021$ $0.85[0.47,1.55] 2021$

$0.83[0.60,1.16]$

Test for overall effect: $Z=1.07(P=0.28)$

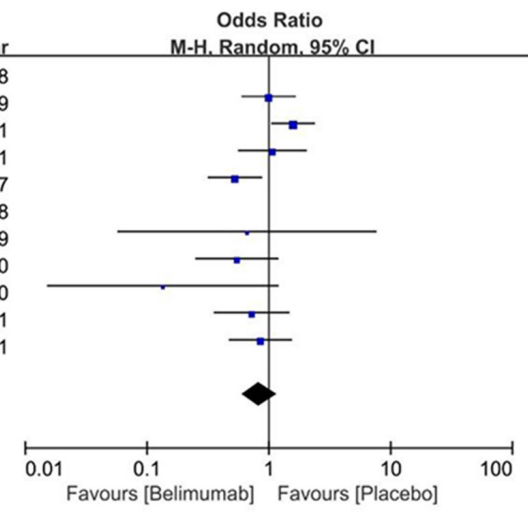

C

\begin{tabular}{|c|c|c|c|c|c|}
\hline \multirow[b]{2}{*}{ Study or Subgroup } & \multicolumn{2}{|c|}{ Belimumab } & \multicolumn{2}{|c|}{ Placebo } & \multirow[b]{2}{*}{ Weight } \\
\hline & Events & Total & Events & Total & \\
\hline Furie 2008 & 0 & 16 & 0 & 4 & \\
\hline Wallace 2009 & 1 & 336 & 0 & 113 & $1.6 \%$ \\
\hline Navarra 2011 & 1 & 578 & 0 & 287 & $1.5 \%$ \\
\hline Furie 2011 & 0 & 753 & 0 & 381 & \\
\hline Stohl 2017 & 2 & 556 & 0 & 280 & $1.5 \%$ \\
\hline Zhang 2018 & 1 & 451 & 1 & 226 & $2.9 \%$ \\
\hline Bae 2019 & 0 & 29 & 0 & 39 & \\
\hline Furie 2020 & 7 & 448 & 12 & 448 & $26.2 \%$ \\
\hline Brunner 2020 & 0 & 53 & 3 & 40 & $8.7 \%$ \\
\hline Ginzler 2021 & 0 & 331 & 2 & 165 & $7.4 \%$ \\
\hline Sheikh 2021 & 28 & 2001 & 23 & 2002 & $50.2 \%$ \\
\hline Total $(95 \% \mathrm{Cl})$ & & 5552 & & 3985 & $100.0 \%$ \\
\hline Total events & 40 & & 41 & & \\
\hline Heterogeneity: $\mathrm{Chi}^{2}=$ & $88, \mathrm{df}=7$ & $(P=0$ & 44); $1^{2}=$ & & \\
\hline
\end{tabular}

Odds Ratio . Fixed. $95 \% \mathrm{Cl}$ Year Not estimable 2008 $1.01[0.04,25.09] 2009$ $1.49[0.06,36.78] 2011$ Not estimable 2011 $2.53[0.12,52.86] 2017$ $0.50[0.03,8.03] 2018$ Not estimable 2019 $0.58[0.22,1.48] 2020$ $0.10[0.01,2.00] 2020$ $0.10[0.00,2.07] 2021$ $1.22[0.70,2.13] 2021$

$0.87[0.57,1.33]$

Test for overall effect: $Z=0.64(P=0.52)$

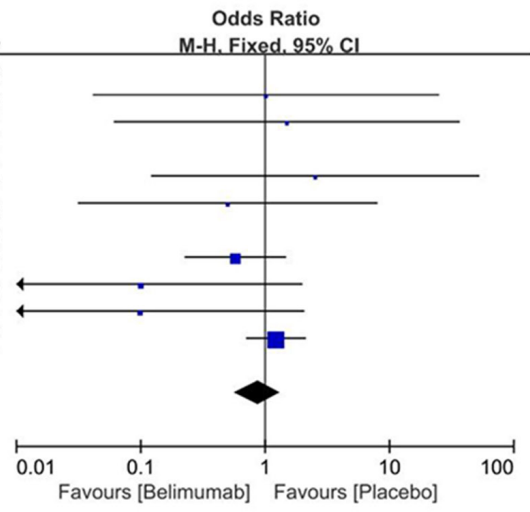

D

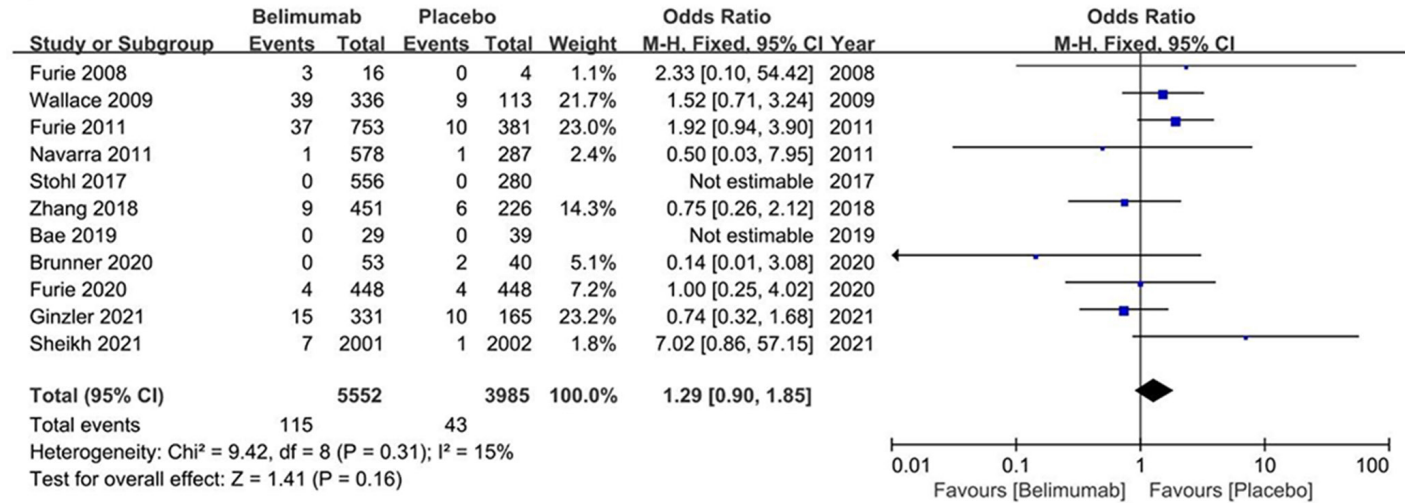

Figure 4 OR of psychiatric events in patients with SLE receiving belimumab compared with placebo in randomised controlled trials: (A) serious psychiatric disorders, (B) non-serious psychiatric disorders, (C) suicidal ideation or behaviour, and (D) depression. 


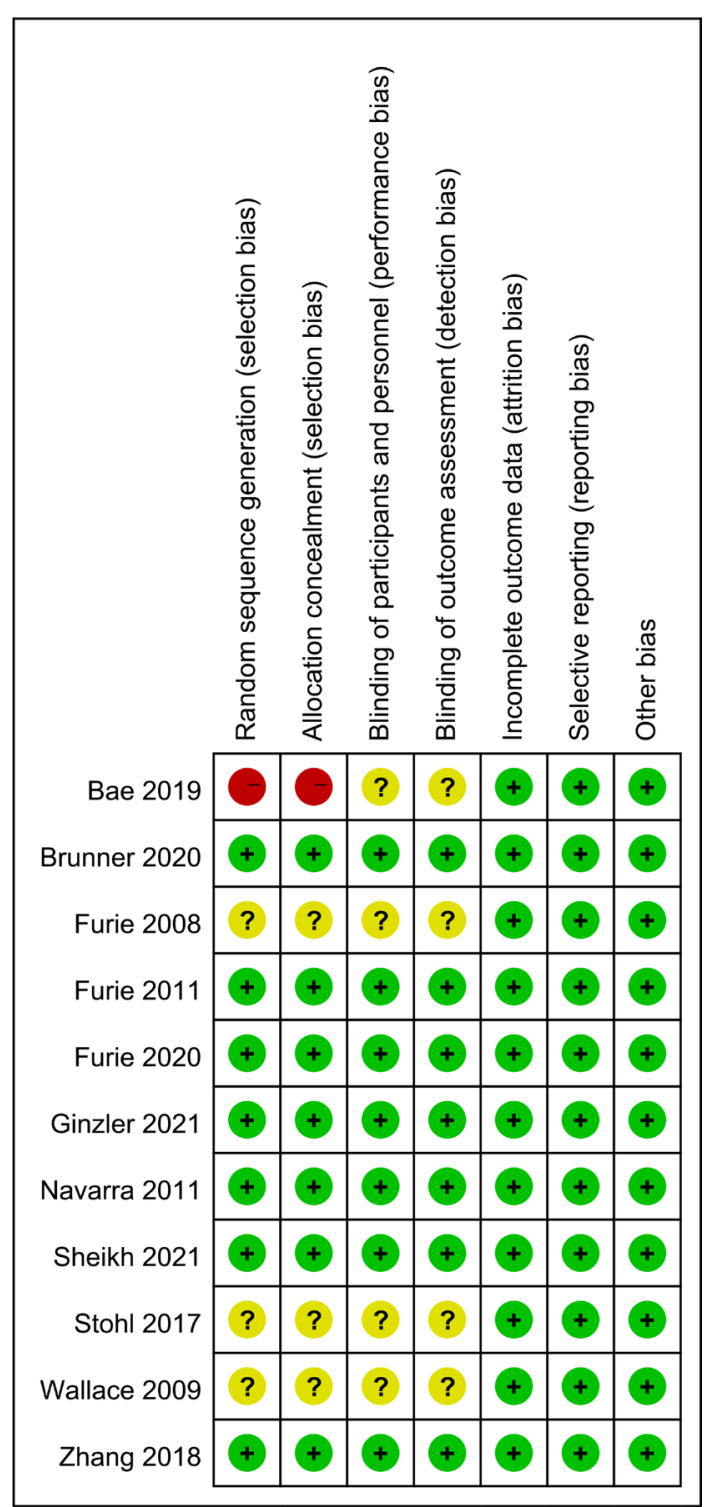

Figure 5 Risk of bias assessment for randomised controlled trials.

be helpful for physicians to identify the patients at risk of developing serious psychiatric adverse events. At present, we may need to assess the psychiatric risk (eg, history of mental disorders) before initiating belimumab therapy, and rheumatologists should seek advice from a psychiatrist if clinically necessary. Additionally, considering the increased risk of psychiatric disorders in patients with SLE and difficulties in diagnosing or excluding neuropsychiatric lupus even in randomised trials, an independent psychiatric safety endpoint adjudication committee reviewing all potential psychiatric events should be routinely established in future clinical trials. At present, special caution is needed when initiating belimumab therapy in patients with existing mental problems, such as depression, suicidal ideation or behaviour until more information becomes available.

We are aware of the major limitation of our metaanalysis. The rarity of serious psychiatric disorders may be inadequately addressed in the context of limited observation duration in RCT. Although both Mantel-Haenszel and Peto methods were applied, which for sure strengthened the statistical power, the low frequency of psychiatric events in relatively short placebo-controlled period of included trials precluded us from a definite conclusion. Moreover, the possibility of bias in the selection of patients in RCTs could not be excluded, and therefore, the findings may not be generalisable to the real-world population.

In summary, based on the best available evidence from RCTs, the present meta-analysis indicates belimumab therapy in general dose not increase the risk of psychiatric events and all-cause mortality. Continuous postmarketing surveillance is imperative to comprehensively clarify the psychiatric effect of belimumab therapy in patients with SLE, especially serious mental disorders, because BASE Study suggested increased risk of psychiatric adverse events associated with belimumab exposure.

Contributors $\mathrm{ZZ}$ is the guarantor of this article who conceived the study, participated in its design and coordination, and critically revised the manuscript. WX had full access to all the data collection, analysis and interpretation, and drafted the manuscript. HH contributed to the data collection as a study investigator. SZ provided helpful assistance of statistical information. All authors read and approved the final manuscript.

Funding The authors have not declared a specific grant for this research from any funding agency in the public, commercial or not-for-profit sectors.

Competing interests None declared.

Patient consent for publication Not required.

Provenance and peer review Not commissioned; externally peer reviewed. Data availability statement All data relevant to the study are included in the article or uploaded as supplemental information.

Supplemental material This content has been supplied by the author(s). It has not been vetted by BMJ Publishing Group Limited (BMJ) and may not have been peer-reviewed. Any opinions or recommendations discussed are solely those of the author(s) and are not endorsed by BMJ. BMJ disclaims all liability and responsibility arising from any reliance placed on the content. Where the content includes any translated material, BMJ does not warrant the accuracy and reliability of the translations (including but not limited to local regulations, clinical guidelines, terminology, drug names and drug dosages), and is not responsible for any error and/or omissions arising from translation and adaptation or otherwise.

Open access This is an open access article distributed in accordance with the Creative Commons Attribution Non Commercial (CC BY-NC 4.0) license, which permits others to distribute, remix, adapt, build upon this work non-commercially, and license their derivative works on different terms, provided the original work is properly cited, appropriate credit is given, any changes made indicated, and the use is non-commercial. See: http://creativecommons.org/licenses/by-nc/4.0/.

ORCID iD

Wenhui Xie http://orcid.org/0000-0002-3881-0266

\section{REFERENCES}

1 Singh JA, Shah NP, Mudano AS. Belimumab for systemic lupus erythematosus. Cochrane Database Syst Rev 2021;2:CD010668.

2 Kaegi C, Steiner UC, Wuest B, et al. Systematic review of safety and efficacy of belimumab in treating immune-mediated disorders. Allergy 2021;76:2673-83.

3 Furie R, Rovin BH, Houssiau F, et al. Two-Year, randomized, controlled trial of belimumab in lupus nephritis. $N$ Engl J Med 2020;383:1117-28.

4 Sheikh SZ, Scheinberg MA, Wei JC-C, et al. Mortality and adverse events of special interest with intravenous belimumab for adults with active, autoantibody-positive systemic lupus erythematosus (base): a 
multicentre, double-blind, randomised, placebo-controlled, phase 4 trial. Lancet Rheumatol 2021;3:e122-30.

5 Tanaka Y, Bae S-C, Bass D, et al. Long-term open-label continuation study of the safety and efficacy of belimumab for up to 7 years in patients with systemic lupus erythematosus from Japan and South Korea. RMD Open 2021;7:e001629.

6 Moher D, Liberati A, Tetzlaff J, et al. Preferred reporting items for systematic reviews and meta-analyses: the PRISMA statement. BMJ 2009;339:b2535.

7 Higgins JP, Altman DG. Assessing risk of bias in included studies. In: Higgins JPT, Green S, eds. Cochrane Handbook for systematic reviews of interventions. Chichester: John Wiley \& Sons Ltd, 2008: 187-242.

8 Furie R, Stohl W, Ginzler EM, et al. Biologic activity and safety of belimumab, a neutralizing anti-B-lymphocyte stimulator (BLyS) monoclonal antibody: a phase I trial in patients with systemic lupus erythematosus. Arthritis Res Ther 2008;10:R109.

9 Wallace DJ, Stohl W, Furie RA, et al. A phase II, randomized, doubleblind, placebo-controlled, dose-ranging study of belimumab in patients with active systemic lupus erythematosus. Arthritis Rheum 2009;61:1168-78.

10 Navarra SV, Guzmán RM, Gallacher AE, et al. Efficacy and safety of belimumab in patients with active systemic lupus erythematosus: a randomised, placebo-controlled, phase 3 trial. Lancet 2011;377:721-31.

11 Furie R, Petri M, Zamani O, et al. A phase III, randomized, placebocontrolled study of belimumab, a monoclonal antibody that inhibits B lymphocyte stimulator, in patients with systemic lupus erythematosus. Arthritis Rheum 2011;63:3918-30.

12 Stohl W, Schwarting A, Okada M, et al. Efficacy and safety of subcutaneous belimumab in systemic lupus erythematosus: a FiftyTwo-Week randomized, double-blind, placebo-controlled study. Arthritis Rheumatol 2017;69:1016-27.

13 Zhang F, Bae S-C, Bass D, et al. A pivotal phase III, randomised, placebo-controlled study of belimumab in patients with systemic lupus erythematosus located in China, Japan and South Korea. Ann Rheum Dis 2018;77:355-63.

14 Brunner HI, Abud-Mendoza C, Viola DO, et al. Safety and efficacy of intravenous belimumab in children with systemic lupus erythematosus: results from a randomised, placebo-controlled trial. Ann Rheum Dis 2020;79:1340-8.

15 Ginzler E, Guedes Barbosa LS, D'Cruz D, et al. Embrace: phase $3 / 4$, randomized, 52 -Week study of belimumab efficacy and safety in patients of black African ancestry with systemic lupus erythematosus. Arthritis Rheumatol 2021.

16 Bae S, Dimelow R, Ji B. Results of the open-label, Non-randomized 52-week study to evaluate treatment holidays and rebound phenomenon after treatment with belimumab in patients with SLE. Arthritis Rheumatol 2019;71:4510-2.

17 Wise LM, Stohl W. The safety of belimumab for the treatment of systemic lupus erythematosus. Expert Opin Drug Saf 2019;18:1133-44. 